\title{
Symmetry breaking of PT-symmetric solitons in self-defocusing saturable nonlinear Schrödinger equation
}

\author{
Wen-Bo Bo ${ }^{1}$, Yue-Yue Wang ${ }^{1, *}$, Peng-Fei $\mathrm{Li}^{2, *}$ and Chao-Qing Dai ${ }^{1, *}$ \\ ${ }^{1}$ College of Optical, Mechanical and Electrical Engineering, Zhejiang A\&F University, \\ Lin'an 311300, China \\ ${ }^{2}$ Department of Physics, Taiyuan Normal University, Jinzhong, 030619, China
}

\begin{abstract}
The symmetry breaking phenomenon of the parity-time (PT) symmetric solitons in selfdefocusing saturable nonlinear Schrödinger equation is studied. As the soliton power increases, branches of asymmetric solitons are separated from antisymmetric solitons, and they coexist with both symmetric and antisymmetric solitons. The anti-symmetric solitons require different power thresholds when they are under different saturable nonlinear strength. The stronger the saturable nonlinearity is, the larger the power threshold is. The saturable nonlinear strength has obvious modulation effect on the symmetry breaking of antisymmetric solitons and the bifurcation of the power curve. However, when the modulation strength of PT- symmetric potential increases, the effect of this modulation effect weakens. The antisymmetric solitons are only stable in the low power region, and the stability of symmetric and asymmetric solitons is less affected by the soliton power. The increase of the saturable nonlinear strength leads to the increase of the critical power of the symmetry breaking. When a beam propagates in a PT-symmetric optical waveguide, the symmetry breaking of antisymmetric solitons can be controlled by changing the saturable nonlinear strength.
\end{abstract}

Keywords. Symmetry breaking; Nonlinear Schrödinger equation; Parity-time symmetry; Saturable nonlinearity; Solitons

\section{Introduction}

Symmetry breaking phenomenon in nonlinear quantum systems is a basic phenomenon attributed to the interaction of nonlinear and symmetric linear potentials [1]. Symmetry breaking has been extensively studied in Bose-Einstein condensates [5-7], lasers [8], liquid crystals [9], etc. In nonlinear optics, with the increase of power, one or more asymmetrical states appear in the system, namely, the phenomenon of the symmetry breaking [2-4], which has been reported successively in thin film optical mechanics [10], moving medium [11], laser dynamics [12] and one-dimensional PT- symmetric optical potential [13].

In the past few years, soliton dynamics in the PT-symmetric potential has been studied extensively both theoretically and experimentally. The eigen spectrum of PT-symmetric non-Hermitian Hamiltonians with pure real numbers was first proposed by Bender and Boettcher in 1998. The gain and loss of a PT-symmetric system are balanced, and the PT symmetry is rooted in the nonHermitian quantum mechanics with PT-symmetric potential [14]. Subsequently, the concept of PT symmetry was extended to optics $[15,16]$. There are a number of typical features in PT-symmetric system, such as the real eigen spectrum $[14,17,18]$ and the existence of a continuous family of solitons [19-21]. These characteristics show that the PT-symmetric system is different from the

*Corresponding author email: wangyy424@163.com (Y.Y.Wang); lpf281888@gmail.com (P.F. Li); dcq424@126.com (C.Q. Dai) 
general dissipative system. It has the property of a conservative system before the occurrence of the symmetry breaking, and shows the property of a dissipative system after the occurrence of the symmetry breaking. Therefore, the PT-symmetric system establishes the relationship between the conservative system and the dissipative system. The necessary condition for PT-symmetric potential is $U(x)=U^{*}(-x)$ where $x$ is the normalized horizontal coordinate, “* "denotes complex conjugate. Thus, the imaginary part of $U(x)$ requires odd function and the real part of $U(x)$ requires even function

In nonlinear optical waveguides, the transmission of the paraxial ray can be described by the nonlinear Schrödinger equation (NLSE) $[23,24]$. The refractive index distribution of the optical waveguide is described by the real potential function, while the loss and gain in the optical waveguide are represented by the imaginary potential function [16,17,22]. Recently, the asymmetric solitons in the PT-symmetric potential have attracted more and more attention. Because the symmetry of solitons is broken, their contours become asymmetric. Perturbation analysis shows that in the PT-symmetric potential, such symmetry breaking needs to satisfy an infinite number of nontrivial conditions simultaneously, so it is impossible to occur in the general PT-symmetric potential [25]. But in a special type of PT-symmetric potential, there are stable PT-symmetry breaking solitons [13]. It is proved that the symmetry breaking of solitons exist in a special type of PT-symmetric potential.

Recently, the symmetry breaking of the soliton in the PT-symmetric potential were intensely investigated. The symmetry breaking of solitons was found in PT-symmetric optical waveguides with focused saturable nonlinearity [26]. And the existence and stability of solitons in PT-symmetric optical lattices in fractional NLSE models were studied [27]. Subsequently, the fork bifurcation of the symmetry breaking for fractional optical solitons were reported [28], and the symmetry breaking behavior of solitons were found in partial PT-symmetric potential [29]. The soliton solutions of the $(1+1)$ dimensional nonhomogeneous cubic-quintic-septimal NLSE with PT-symmetric potential were discussed [30]. Then the symmetric-broken and symmetric-unbroken soliton solutions of the semi-discrete nonlocal NLSE were also discussed [31]. In addition, the phenomena of dark solitons and vortices from spontaneous symmetry breaking to nonlinear PT phase transition were found in PT-symmetric nonlinear system [32]. Although there have been many studies on the NLSEs under the PT-symmetric potential and, the solitons of the NLSE supported by the PT-symmetric potential and the saturable defocusing nonlinearity is less studied.

In previous studies, the saturable nonlinear strength and the shape and parameters of the potential function have certain effects on the symmetry breaking of the soliton under the PTsymmetric potential. But the modulating relationship between them has never been discussed. In this paper, the symmetry breaking of solitons is studied based on the self-defocusing saturable NLSE, and the effects of symmetry breaking and bifurcation of solitons are analyzed in detail. Furthermore, the relationship among the modulating strength of the PT-symmetric potential, the modulation strength of PT-symmetric potential, the saturable nonlinear strength and the power threshold leading 
to the symmetry breaking is explored. It is found that when the modulation strength of PTsymmetric potential function reaches a certain threshold, the power threshold leading to the symmetry breaking will no longer change with the add of saturable nonlinear strength. Beyond the modulation threshold of potential function, saturable nonlinearity can no longer modulate symmetry breaking. This provides a new way to control the transformation of antisymmetric solitons into asymmetric solitons in the PT-symmetric system.

\section{Model}

In the approximately paraxial conditions, the propagation of optical wave in a saturable nonlinear waveguide is represented by the following normalized NLSE [29]

$$
i \frac{\partial \psi}{\partial z}+\frac{\partial^{2} \psi}{\partial x^{2}}+U(x) \psi+\frac{\sigma|\psi|^{2} \psi}{1+S|\psi|^{2}}=0
$$

where $\psi(z, x)$ represents the distribution of the light field and $U(x) \equiv V R(x)+i V I(x)$ is the normalized PT-symmetric potential, $\sigma=+1$ represents the focusing nonlinearity and $\sigma=-1$ represents selfdefocus nonlinearity, the parameter $S$ defines the nonlinear saturable. When the self-defocusing nonlinear parameter $\sigma=0$, Eq. (1) gives a description of the PT-symmetric system. In this paper, we consider the self-defocusing saturable nonlinearity with $\sigma=-1$.

In order to explore the symmetry breaking phenomenon of solitons in Eq.(1), the form of the steady-state solution is expressed as $\psi(z, x)=\phi(x) e^{i \beta z}$, where $\phi(x)$ contains the real part and the imaginary part which are $\phi_{R}$ and $\phi_{I}$. The propagation constant is described by the real value $\beta$. Substituting the steady-state solution into Eq. (1) leads to

$$
\frac{d^{2} \phi}{d x^{2}} \phi(x)+U(x) \phi(x)+\sigma \frac{|\phi|^{2} \phi}{1+S|\phi|^{2}}=\beta \phi(x)
$$

In our research, we select the following PT-symmetric potential function [29] as

$$
U(x)=u^{2}(x)+i \frac{d u(x)}{d x}
$$

where $u(x)$ is a real even symmetric function. We choose the form of $u(x)$ as

$$
u(x)=\omega_{0}\left[\operatorname{sech}\left(\frac{x+x_{0}}{\kappa_{0}}\right)+\operatorname{sech}\left(\frac{x-x_{0}}{\kappa_{0}}\right)\right]
$$

where $\omega_{0}$ describes the modulation strength of the PT-symmetric potential, $x_{0}$ represents the distance between the two peaks of the potential function, and $\kappa_{0}$ represents the width of a single peak of the potential function. Thus, the real and imaginary parts of PT-symmetric potential are respectively

$$
\begin{gathered}
V R(x)=\omega_{0}^{2}\left[\operatorname{sech}\left(\frac{x+x_{0}}{\kappa_{0}}\right)+\operatorname{sech}\left(\frac{x-x_{0}}{\kappa_{0}}\right)\right]^{2} \\
V I(x)=-\frac{\omega_{0}}{\lambda_{0}}\left[\operatorname{sech}\left(\frac{x+x_{0}}{\kappa_{0}}\right) \tan \left(\frac{x+x_{0}}{\kappa_{0}}\right)+\operatorname{sech}\left(\frac{x-x_{0}}{\kappa_{0}}\right) \tan \left(\frac{x-x_{0}}{\kappa_{0}}\right)\right]
\end{gathered}
$$

\section{The numerical results}


We aim to find the symmetric, asymmetric and antisymmetric soliton solutions, and explore the corresponding symmetry breaking phenomenon.

First, we study the self-defocus strongly saturable nonlinearity $S=1$. The function $u(x)$, and two parts of the PT- symmetric potential function, $V R(x)$ and $V I(x)$, are shown in Fig. 1(a).

(a)

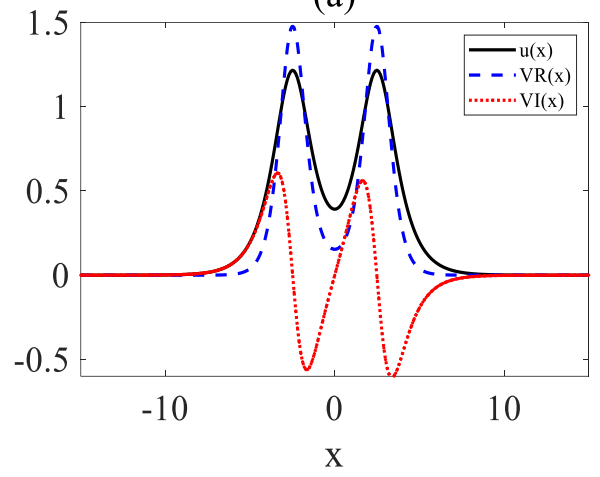

(c)

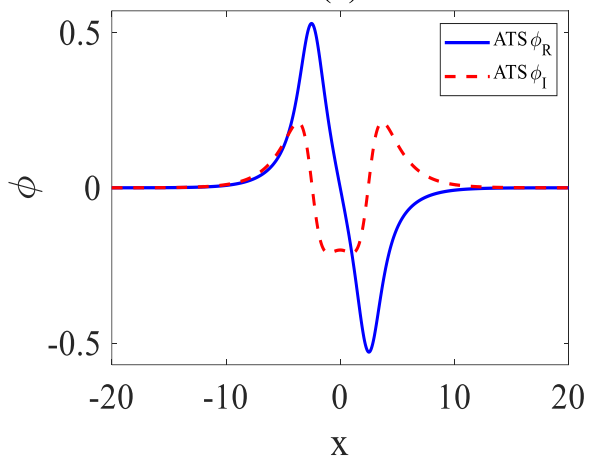

(b)

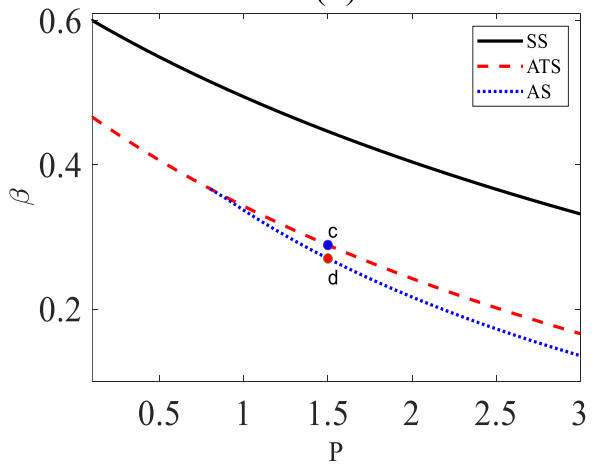

(d)

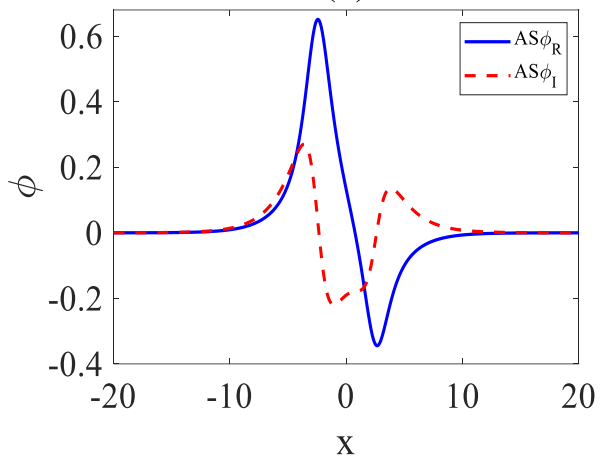

Figure 1. PT-symmetric potential function, antisymmetric and asymmetric solitons and symmetry breaking bifurcation. (a) the real even symmetric function $u(x)$ (black solid curve), the real part (blue dashed curve) and the imaginary part (red blue dashed curve) of the potential function. (b) The power of symmetric solitons (black solid curve marked SS), asymmetric solitons (blue dashed curve marked AS), and antisymmetric solitons (red dashed curve marked ATS) v.s. the propagation constant. The real part (blue solid curve) and imaginary part (red dashed curve) of (c) antisymmetric and (d) asymmetric solitons with the soliton power $P=1.5$. Here, parameters are $x_{0}=2.5, \lambda_{0}=1.0$, $\omega_{0}=1.2$.

These steady-state solutions can be obtained by using the Power-conserving square operator method [24], in which the soliton power is defined as

$$
P(\beta)=\int_{-\infty}^{+\infty}|\psi(x ; \beta)|^{2} d x
$$

The numerical results show that there are two kinds of soliton solutions, namely PT-symmetric soliton solutions and non-PT-symmetric soliton solutions in Eq. (2). The bifurcation diagram of the power curve in Fig. 1(b) shows that symmetric soliton solutions, antisymmetric soliton solutions and asymmetric soliton solutions all exist in the case of self-defocusing saturable nonlinearity. From Fig. 1(b), with the improve of power, new asymmetric solitons are generated and separated from antisymmetric solitons at $P=0.8$. Beyond the critical point, the symmetry of the antisymmetric solitons is broken, but the symmetry of the symmetric solitons maintains. In a conservative system, 
if the slopes of antisymmetric and asymmetric solitons have same signs at bifurcation points, then antisymmetric and asymmetric solitons will have opposite stability or instability [33], which is similar to the results from the linear stability analysis and dynamic evolution in Figs. 5 and 6. We can see the real and imaginary parts of the antisymmetric and asymmetric solitons in Fig. 1(c) and Fig. 1(d) (corresponding to points $\mathrm{c}$ and d in Fig. 1(b)), respectively. The real and imaginary parts of antisymmetric solitons are odd symmetric and even symmetric respectively, while the real and imaginary parts of asymmetrical solitons are both asymmetric.

(a)

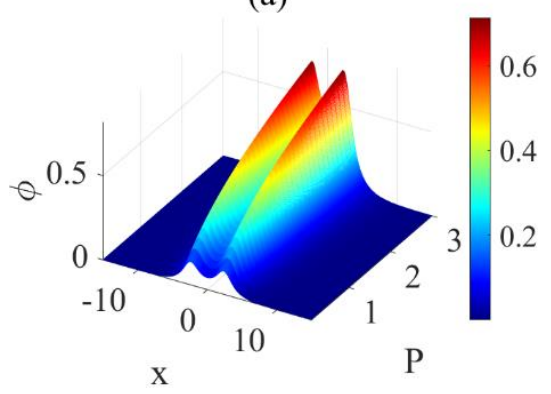

(b)

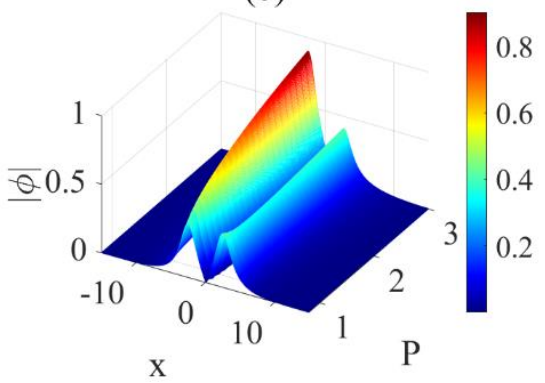

$\mathrm{x}$

(c)

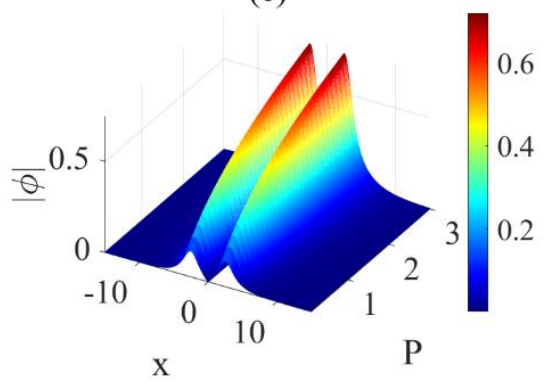

Figure 2. (a) Symmetric soliton, (b) asymmetric soliton, and (c) antisymmetric soliton at different powers. Parameters are $x_{0}=2.5, \lambda_{0}=1.0, \omega_{0}=1.2$.

The profiles of symmetric, asymmetric, and antisymmetric solitons with varying power are shown in Figure 2. In Fig.2(b), asymmetric soliton exists between $P=0.8$ and $P=3$. As the power increases, the energy is gradually concentrated on one of the peaks.

(a)

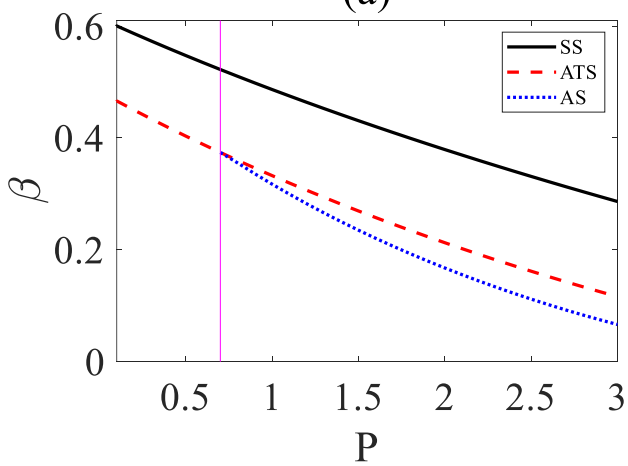

(b)

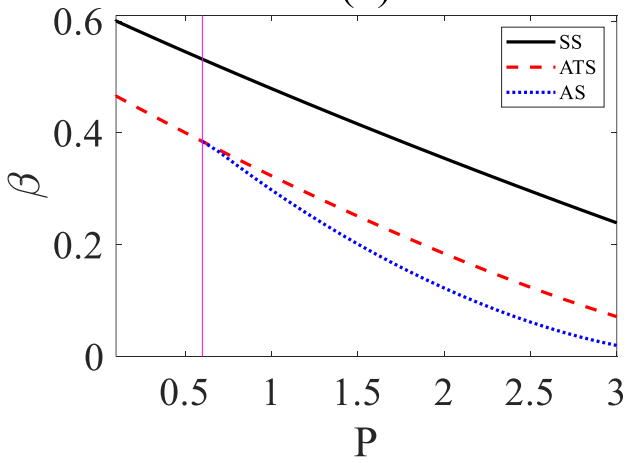

Figure 3. Bifurcation diagrams of symmetric solitons (black solid curve marked SS), asymmetric solitons (blue dashed curve marked AS), and antisymmetric solitons (red dashed curve marked ATS) 
for moderate and weak saturable nonlinear strength with (a) $S=0.5$ and (b) $S=0.1$. The positions of bifurcation points are indicated by solid purple lines. Parameters are $x_{0}=2.5, \lambda_{0}=1.0, \omega_{0}=1.2$.

Next, the influence of saturable nonlinear parameters on the soliton bifurcation is discussed for different saturable nonlinear parameters. The soliton bifurcations for strongly weakly, moderately and weakly saturable nonlinearities are given in Fig.1(b)、Figs.3(b) 、Figs.3 (c), respectively. As shown in Fig. 1(b), under the condition of strongly saturable nonlinearity ( $S=1$ ), when the soliton power exceeds 0.8 , asymmetric soliton is separated from antisymmetric solitons, the symmetry breaking phenomenon occurs at the same time, and the corresponding bifurcation of powerpropagation constant is generated. Figs. 3(a) and 3(b) show that the power required to generate the symmetry breaking is $P=0.7$ and $P=0.6$ respectively, when the saturable nonlinear strength is moderate ( $S=0.5$ ) and the saturable nonlinear strength is weak $(S=0.1)$.These numerical results show that different saturable nonlinear strengths can change the soliton power required for the symmetry breaking.

At last, we will explore the influence of the modulation strength $\omega_{0}$ of the PT-symmetric potential function on the symmetry breaking. Figs.4(a)-(c),(d)-(f),(g)-(i) show the breaking bifurcation phenomenon under different saturable nonlinear strength sand the modulation strength $\omega_{0}=1.2, \omega_{0}=1.3, \omega_{0}=1.5$, respectively. The powers of bifurcation points in Figs. 4(a)-(i) are $P=0.8$, $0.7,0.6,0.5,0.4,0.4,0.2,0.2$ and 0.2 , respectively. According to Fig. 4(a), 4(d) and 4(g), under the same saturable nonlinear strength, the power required to generate symmetry breaking decreases with the increase of modulation strength. This shows that when the modulation strength of potential function is low, higher soliton power is needed to produce the symmetry breaking. The values of the critical power and corresponding propagation constant $P_{c r}\left(\beta_{c r}\right)$ at the bifurcation point in the Figure 4 are listed in Table 1. Figs. 4(a)-(c), for the same modulation strength, with the increase of saturable nonlinear strength, the soliton power required to produce the symmetry breaking phenomenon also increases.

(a)

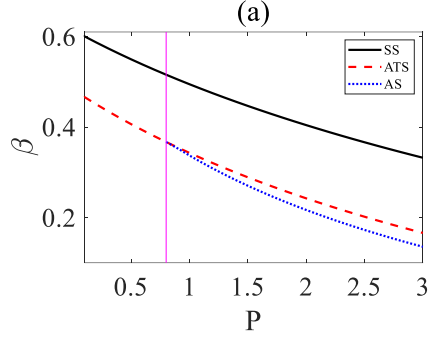

(d)

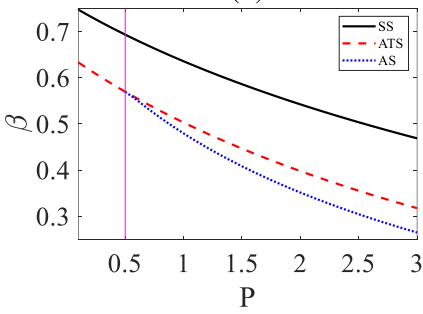

(g)

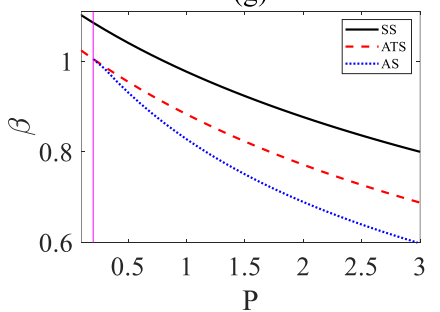

(b)

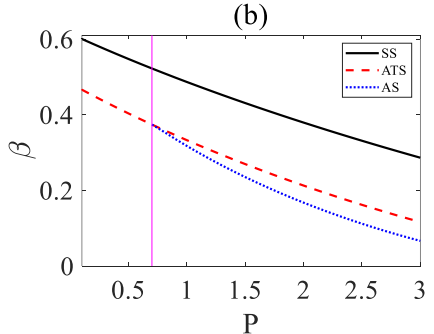

(e)

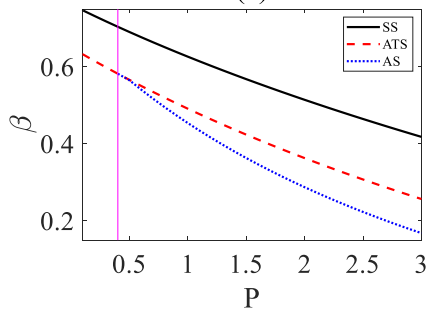

(h)

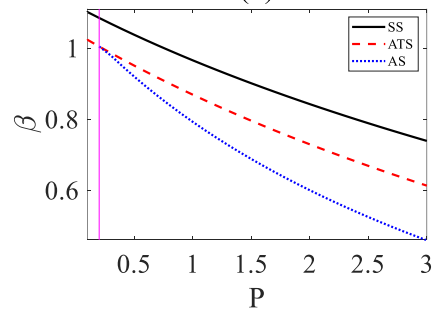

(c)

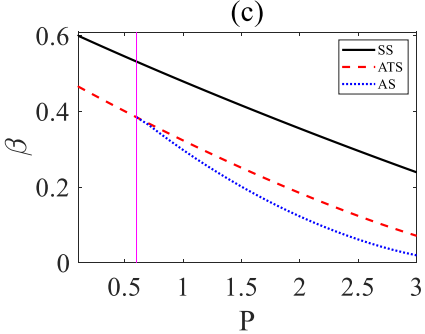

(f)

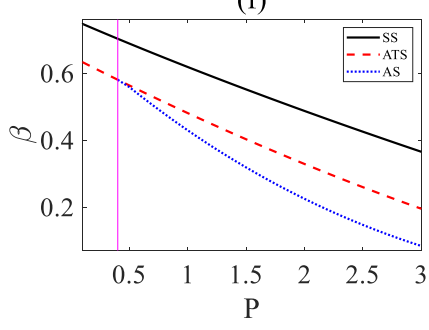

(i)

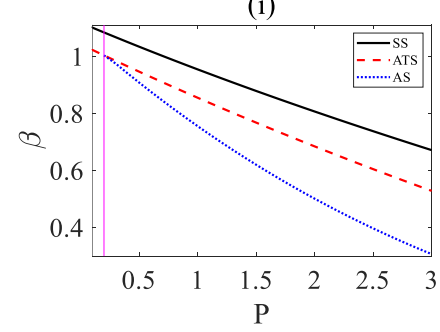


Figure 4.Bifurcation diagrams of symmetric solitons (black solid curve marked SS), asymmetric solitons (blue dashed curve marked AS), and antisymmetric solitons (red dashed curve marked ATS) for different saturable nonlinear parameters with (a)(d)(g) $S=1$, (b)(e)(h) $S=0.5$ and (c)(f)(i) $S=0.1$ and the modulation strength with (a)-(c) $\omega_{0}=1.2$, (d)-(f) $\omega_{0}=1.3$, and (g)-(i) $\omega_{0}=1.5$. The locations of bifurcation points are indicated by purple lines.

Table 1. Values of the critical power and corresponding propagation constant $P_{c r}\left(\beta_{c r}\right)$ at bifurcation points of the symmetry breaking in the self-defocusing saturable nonlinear case

\begin{tabular}{|c|c|c|c|}
\hline$a$ & $\omega_{0}=1.2$ & $\omega_{0}=1.3$ & $\omega_{0}=1.5$ \\
\hline$S=1$ & $0.80(0.3669)$ & $0.50(0.5697)$ & $0.20(1.0053)$ \\
\hline$S=0.5$ & $0.70(0.3740)$ & $0.40(0.5822)$ & $0.20(1.0046)$ \\
\hline$S=0.1$ & $0.60(0.3848)$ & $0.40(0.5802)$ & $0.20(1.0039)$ \\
\hline
\end{tabular}

In Figs. 4(a)-(c), for the same modulation strength, with the increase of saturable nonlinear strength, the soliton power required to produce the symmetry breaking phenomenon also increases. But does this rule always exist? From the bifurcation images of the symmetry breaking in Figs.4(d)(f) and Fig.4(g)-(i) with the modulation strength $\omega_{0}=1.3$ and $\omega_{0}=1.5$ and strongly $(S=1)$, moderately ( $S=0.5$ ) and weakly ( $S=0.1$ ) saturable nonlinear strength, when the modulation strength $\omega_{0}$ exceeds a certain threshold, the power required to generate the symmetry breaking will not change with the variation of the saturable nonlinear strength, but maintains a constant value for different saturable nonlinear strengths .

4. Linear stability and dynamics

The linear stability of solitons will be analyzed by adding perturbations to the model as:

$$
\psi(z, x)=e^{i \beta z}\left[\phi(x)+\tau(x) e^{\delta z}+\kappa^{*}(x) e^{\delta^{*} z}\right]
$$

where $\kappa(x)$ and $\tau(x)$ are perturbations.

Introducing Eq. (8) into Eq. (1) and just retaining the linear term of the perturbation term get the linear eigenvalue problem

$$
i\left(\begin{array}{ll}
T_{11} & T_{12} \\
T_{21} & T_{22}
\end{array}\right)\left(\begin{array}{l}
\tau \\
\kappa
\end{array}\right)=\delta\left(\begin{array}{l}
\tau \\
\kappa
\end{array}\right)
$$

where the forms of $T_{11}, T_{12}, T_{21}, T_{22}$ are as follows:

$$
T_{11}=-\beta+\frac{d^{2}}{d x^{2}}+U+\frac{2 \sigma|\phi|^{2}}{1+S|\phi|^{2}}-\frac{S \sigma|\phi|^{4}}{\left(1+S|\phi|^{2}\right)^{2}}
$$




$$
\begin{gathered}
T_{12}=\frac{\sigma \phi^{2}}{1+S|\phi|^{2}}-\frac{S \sigma \phi^{2}|\phi|^{2}}{\left(1+S|\phi|^{2}\right)^{2}} \\
T_{21}=-\frac{\sigma\left(\phi^{*}\right)^{2}}{1+S|\phi|^{2}}+\frac{S \sigma\left(\phi^{*}\right)^{2}|\phi|^{2}}{\left(1+S|\phi|^{2}\right)^{2}} \\
T_{22}=\beta-\frac{d^{2}}{d x^{2}}-U^{*}-\frac{2 \sigma|\psi|^{2}}{1+S|\psi|^{2}}+\frac{S \sigma|\psi|^{4}}{\left(1+S|\psi|^{2}\right)^{2}}
\end{gathered}
$$

with the unstable growth rate $\delta$ - (complex values). The linear stability is represented by unstable growth rate $\delta$. The real and imaginary parts of $\delta$ represent the unstable growth rate of the soliton solution, and the shape oscillation in the soliton transmission process, respectively. When the absolute value of the real part of $\delta$ is zero, the soliton solution is linearly stable. The dependence between solitons power and maximum instability growth rates $\operatorname{Max}\left(\delta_{R}\right)$ is shown in Figure 5.The numerical results of symmetric, asymmetric and antisymmetric solitons under the strongly $(S=1)$, moderately ( $S=0.5$ ) and weakly ( $S=0.1$ ) saturable nonlinear strength in the defocusing case are shown in Figs.5 (a), 5 (b) and 5 (c) respectively, corresponding to Figs. 3(a), 3(b) and 3(c). When the power $P$ is less than 0.8 , the symmetric solitons, the asymmetric solitons and the antisymmetric solitons are all stable. When the power $P$ exceeds 0.8 corresponding to Figure $3(\mathrm{a})$, the asymmetric solitons separated from the antisymmetric soliton, resulting in the symmetry breaking phenomenon and generating the bifurcation phenomenon.

(a)

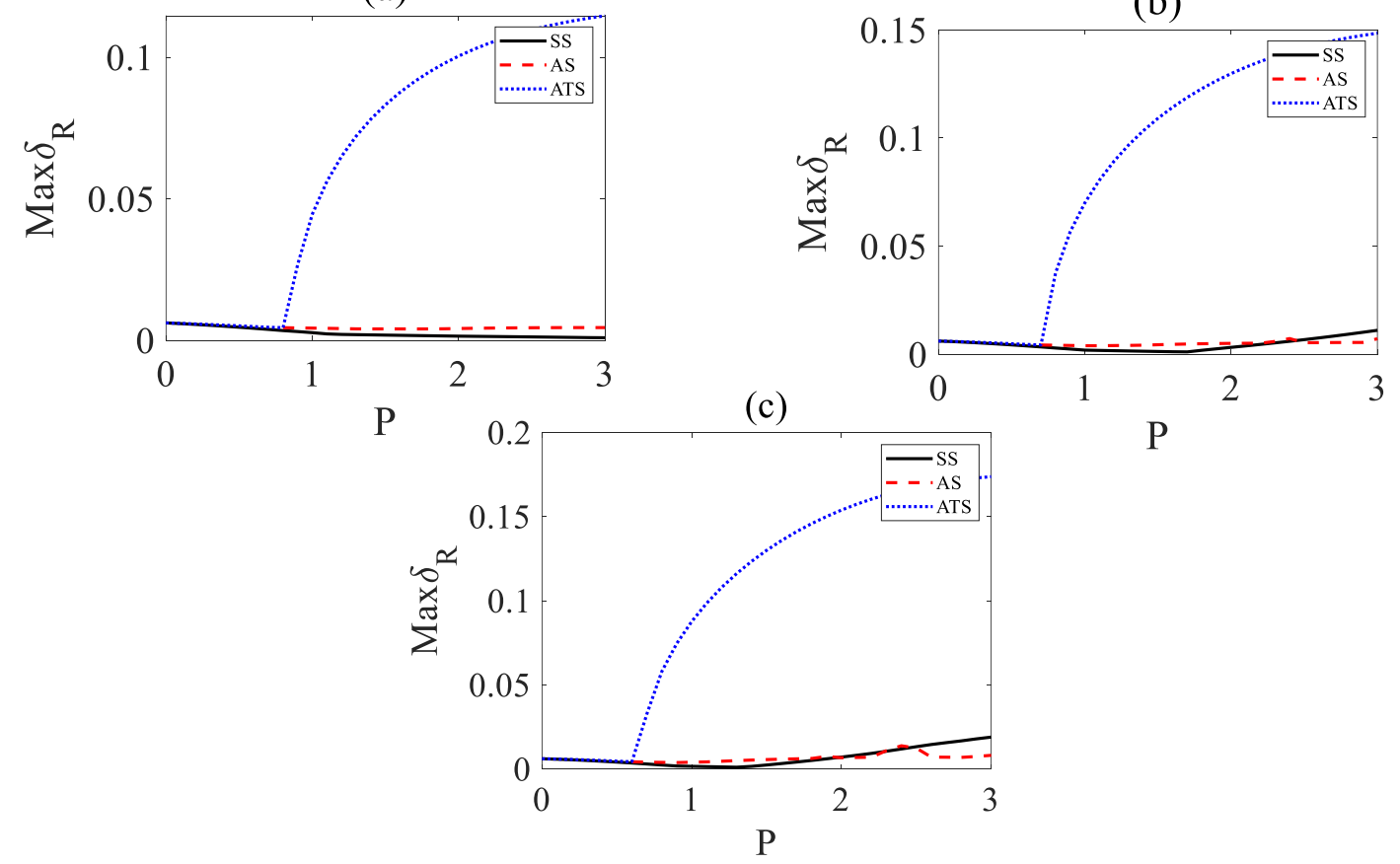

Figure 5. The real part of the maximum unstable growth rate of symmetric solitons (black solid curve marked SS), asymmetric solitons (red dashed curve marked AS), and antisymmetric solitons 
(blue dashed curve marked ATS) with nonlinear parameters (a) $S=1$, (b) $S=0.5$ and (c) $S=0.1$ for the defocusing saturable nonlinear case. Parameters are $x_{0}=2.5, \lambda_{0}=1.0, \omega_{0}=1.2$.

At the same time, the antisymmetric soliton quickly becomes unstable, but symmetric and asymmetric solitons remain stable. As we can see from Fig. 5(a), both symmetric and asymmetric solitons remain weakly stable when the power $P=0$ adds to $P=2.0$. When the power $P=2.0$ adds to $P=3.0$, symmetric soliton becomes more stable while asymmetric soliton remains weakly stable. The similar phenomenon is also existing in Figs. 5(b) and 5(c), and the difference is that when the saturable nonlinear strength decreases, the power value needed to generate the symmetry breaking bifurcation also decreases.

We also find that with the increase of the saturable nonlinear strength, the stable region of the symmetric soliton increases, but that of the asymmetric soliton does not change significantly and the asymmetric soliton remains weakly stable. When the power $P=1.3$ adds to $P=1.7$ for the moderately ( $S=0.5$ )saturable nonlinear strength in Fig. 5(b), and in Fig.5(c) the power $P=1.2$ adds to $P=1.4$ in the weakly ( $S=0.1$ ) saturable nonlinear strength, the symmetric solitons will both become relatively stable states. The increase of saturable nonlinear strength also expands the stability region of antisymmetric solitons.

The outcomes of the linear stability analysis for solitons are also verified by using the immediate numerical simulations of Eq. (1). Linear-stability spectrums of symmetric, asymmetric and antisymmetric solitons is shown in Figs. 6(a), 6(c), and 6(e). It can be seen in Figs. 6(a) and 6(c) that both symmetric and asymmetric solitons are in a weak stable state.

In order to prove the above results, the dynamical evolution behaviors of symmetric and asymmetric solitons will be studied with the $3 \%$ random noise added in the initial soliton solution in Figs. 6(b) and 6(d). The numerical simulation results show that the symmetric soliton with the weak stable state can propagate stably, but the asymmetric soliton will oscillate and break up after propagating a long distance because of its weak instability. The linear-stability spectrum for antisymmetric soliton in Fig. 6(e) indicates that the antisymmetric soliton is in an unstable state at power $P=1.0$. The corresponding evolution diagram is shown in Fig. 6(f). The antisymmetric soliton can propagate stably at the initial stage, and its amplitude oscillation is enhanced due to its instability, and finally the waveform is completely destroyed.
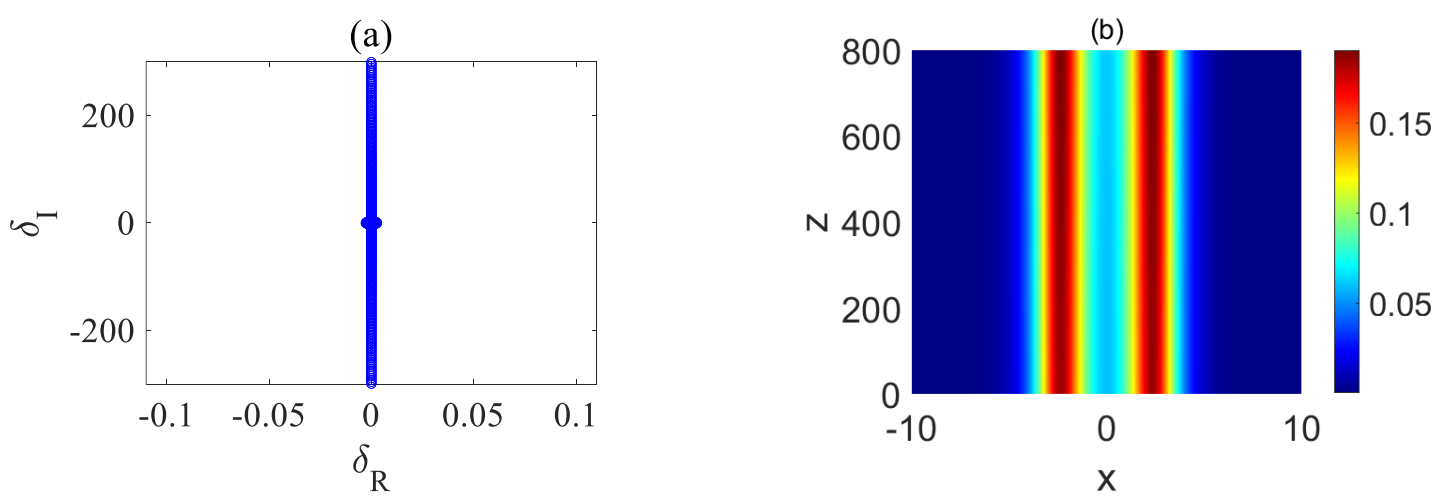
(c)

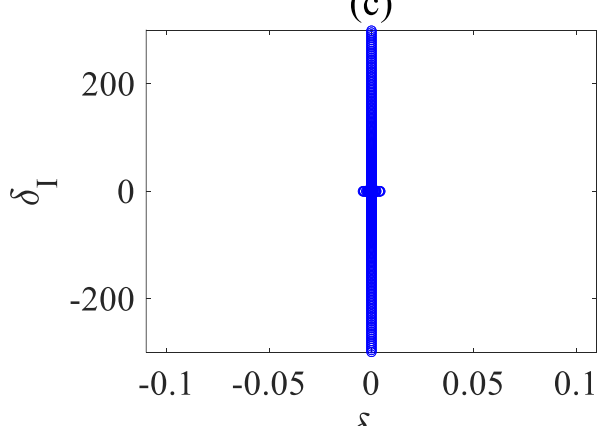

(e)

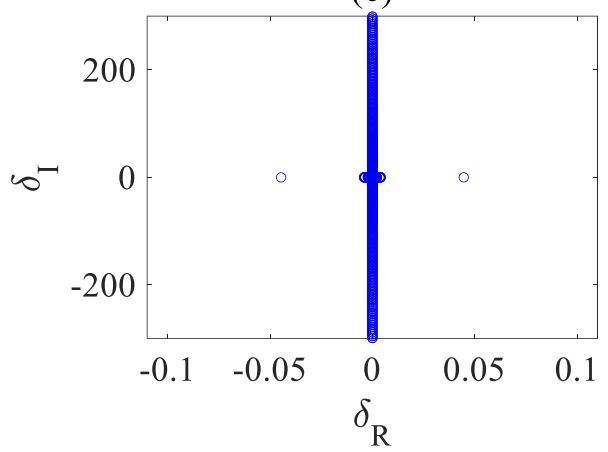

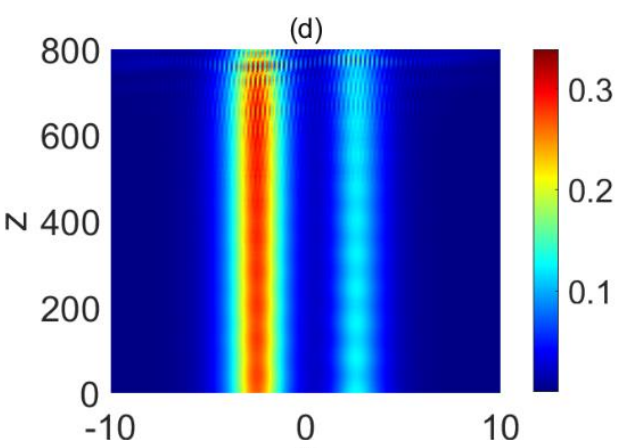

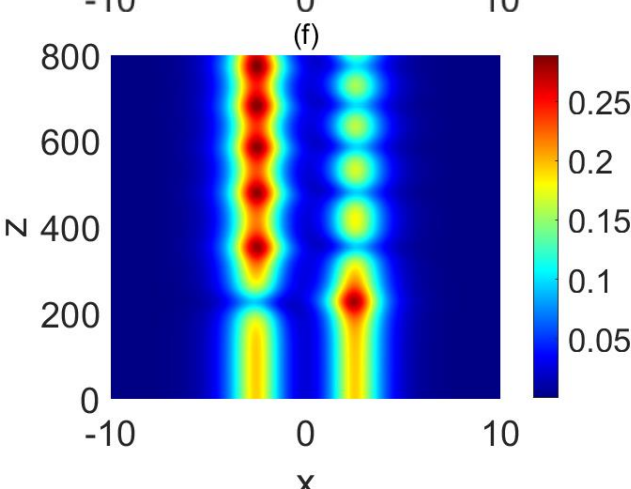

Figure 6. The eigenvalue spectrum of (a) symmetric, (c) asymmetric, and (e) antisymmetric with the strongly saturable nonlinearity $S=1$ and $P=1.0$. (b), (d) and (f) are the diagrams of its evolution respectively. Parameters are $x_{0}=2.5, \lambda_{0}=1.0, \omega_{0}=1.2$.

The linear-stability spectrum and dynamic evolution of solitons from linear stability analysis of for different powers are shown in Figure 7. Here we take the power $P=2.0$ and the strongly saturable nonlinearity $S=1$. The linear-stability spectrums of symmetric, asymmetric and antisymmetric solitons from the linear stability analysis are shown in Figs. 7(a), 7(c), and 7(e). It can be seen that the symmetric soliton at $P=2.0$ in Fig. 7(a) is more stable than the asymmetric soliton in the same case, and both the symmetric and the asymmetric solitons show the weak stability. In order to verify its stability, the initial soliton solution is interfered by $3 \%$ random noise, and the corresponding evolution diagrams are shown in Figs.7 (b) and7 (d). In Fig. 7(b), the symmetric soliton oscillates and ruptures after the stable propagation over a long distance. In Fig. 7(d), the wave of the asymmetric soliton is broken after the stable transmission over a short distance. In Fig. $7(\mathrm{e})$, the linear-stability spectrum of antisymmetric soliton shows that the antisymmetric soliton is in an unstable state when the power $P=2.0$. From the evolution diagram in Fig. 7(f), antisymmetric soliton can only propagate a relatively short distance, and then oscillate and finally lead to waveform rupture.

(a)

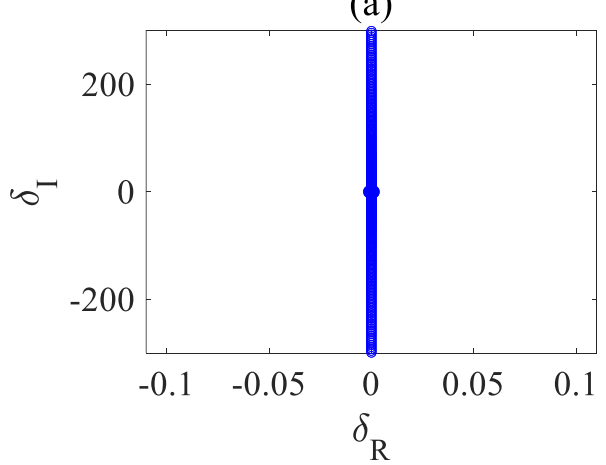

(b)

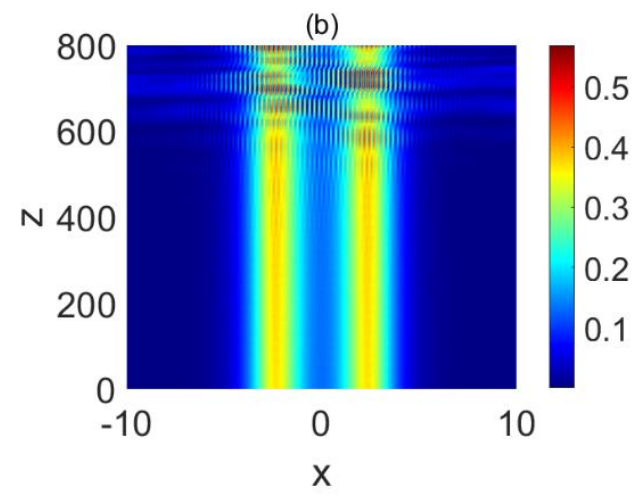


(c)

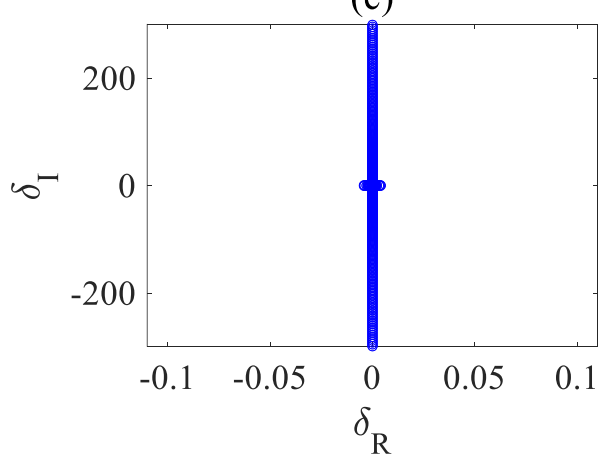

(e)

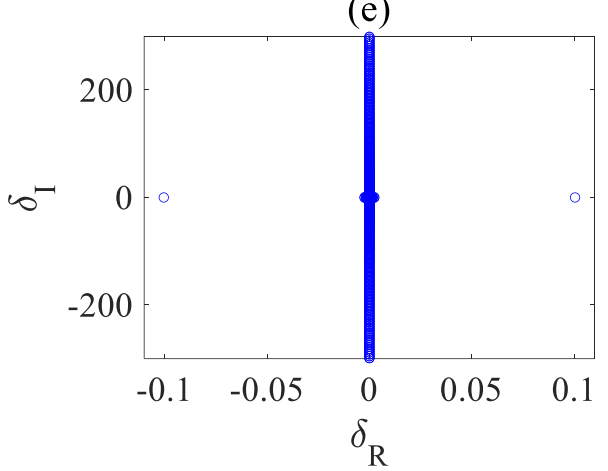

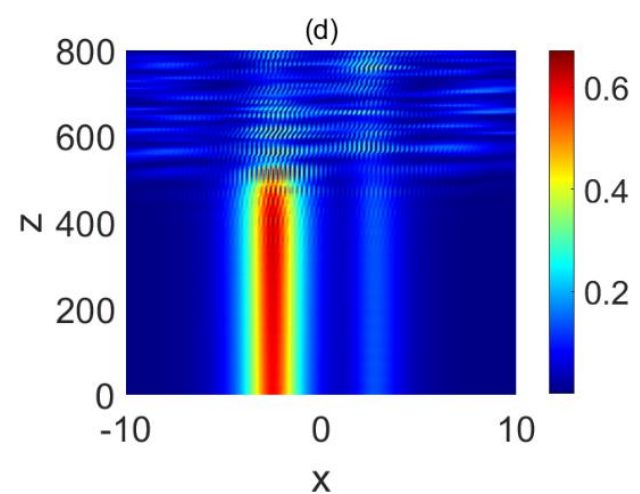

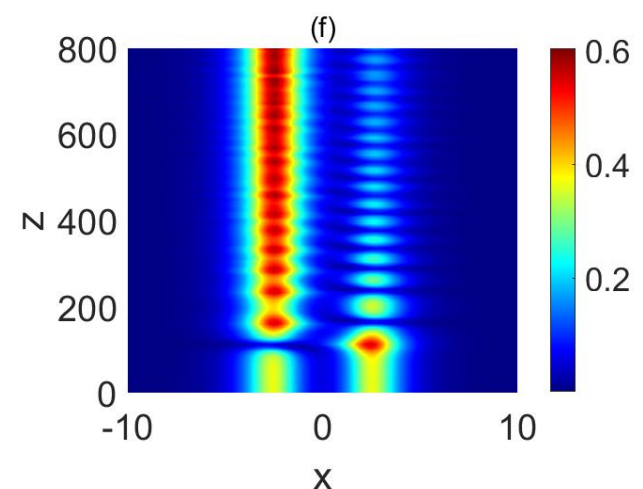

Figure 7. The eigenvalue spectrum of (a) symmetric, (c) asymmetric, and (e) antisymmetric solitons with the strongly saturable nonlinearity $S=1$ and the power $P=2.0$. (b), (d) and (f) the evolution diagrams of solitons corresponding to (a), (c), (e) respectively. Parameters are $x_{0}=2.5, \lambda_{0}=1.0$, $\omega_{0}=1.2$.

\section{Conclusion}

In conclusion, the NLSE with the self-defocus saturable nonlinearity is studied systematically. We prove that the model supports symmetric, asymmetric and antisymmetric solitons by numerical methods. With the increase of soliton power, asymmetric soliton is separated from antisymmetric soliton and coexists with symmetric and antisymmetric solitons.

The power curves of solitons show that the threshold of power required for the symmetry breaking bifurcations generated by different saturable nonlinear strengths is different, and this threshold will not change with the increase of the modulation strength of the potential function. This shows that although the modulation strength $\omega_{0}$ of the potential function can adjust the power threshold leading to the symmetry breaking bifurcations generated by different saturable nonlinear strengths, it has a certain limit in its adjustment capacity, and beyond this limit, the adjustment capacity will be lost. Soliton stability is studied from the linear stability analysis and verified by the direct numerical simulation. Symmetric and asymmetric solitons can propagate stably with the 
change of power, and show a strong robustness. However, antisymmetric soliton will become very unstable and shows a very weak robustness.

\section{Funding}

Zhejiang Provincial Natural Science Foundation of China (Grant No. LR20A050001); National Natural Science Foundation of China (Grant Nos. 12075210 and 11874324); the Scientific Research and Developed Fund of Zhejiang A\&F University (Grant No. 2021FR0009).

\section{Data availability statement}

The datasets generated during and/or analysed during the current study are available from the corresponding author on reasonable request.

\section{Conflict of interest}

The authors have declared that no conflict of interest exists.

\section{Ethical Standards}

This Research does not involve Human Participants and/or Animals.

\section{References}

[1] Malomed, B. A.: Spontaneous symmetry breaking, self-trapping, and Josephson oscillations. Springer Berlin Heidelberg (2013).

[2] Ru, Sin. R., Marangell, R., Susanto, H.: Symmetry breaking bifurcations in the NLS equation with an asymmetric delta potential. Nonlinear Dynamics. 100, 3815-3824 (2020)

[3] Xin, You., Lü, Hui., et al.: PT-Symmetry-Breaking Chaos in Optomechanics. Phys. Rev. Lett. 114, 253601-1-253601-6 (2015)

[4] Xue, yue., Zhang, Qi. Tao., et al.: Symmetry-breaking-induced nonlinear optics at a microcavity surface. Nature Photonics. 13, 21-24 (2019)

[5] Zegadlo, K., Hung, N. V., Konotop, V. V., et al.: Route to chaos in a coupled microresonator system with gain and loss. Nonlinear Dynamics. 97, 559-569 (2019)

[6] Hejazi, S., Polo, J., Sachdeva, R., et al.: Symmetry breaking in binary Bose-Einstein condensates in the presence of an inhomogeneous artificial gauge field. Phys. Rev. A 102, 053309 (2020)

[7] Ikuta, M., Sugano, Y., Saito, H.: Symmetry-breaking instability of leapfrogging vortex rings in a Bose-Einstein condensate. Phys Rev. A 99,043610 (2019).

[8] Kengne, J., Chedjou, J. C., Kom, M., et al.: Regular oscillations, chaos, and multistability in a system of two coupled van der Pol oscillators:numerical and experimental studies. Nonlinear Dynamics. 76, 1119-1132 (2014)

[9] Carsten, T.: Mirror symmetry breaking in liquids and liquid crystals. Liquid Crystals. 45, 2221$2252(2018)$

[10] Ji, Y., Qi, Z., Misra, S., Jin, R., Wang, H.: Breaking Lattice Symmetry in Highly Strained Epitaxial VO 2 Films on Faceted Nanosurface. ACS Applied Materials \& Interfaces. 11, 4490544912 (2019) 
[11] Silveirinha., Mário, G.: Spontaneous Parity-Time Symmetry Breaking in Moving Media. Phys. Rev. A 90, 124-129 (2014)

[12] Ye, Z. J., Chen, Y. X., Zheng, Y. Y., et al.: Symmetry breaking of a matter-wave soliton in a double-well potential formed by spatially confined spin-orbit coupling. Chaos, Solitons \& Fractals. $130,109418(2020)$

[13] Yang, J.: Symmetry breaking of solitons in one-dimensional parity-time-symmetric optical potentials. Opt. Lett. 39, 5547-5550 (2014)

[14] Bender, C.M., Boettcher, S.: Real spectra in non-Hermitian Hamiltonians having PT symmetry. Phys. Rev. Lett. 80, 5243 (1998)

[15] Ruschhaupt, A., Delgado, F., Muga, J. G.: Physical realization of PT-symmetric potential scattering in a planar slab waveguide. Journal of Physics A General Physics. 38, L171 (2017)

[16] Longhi, S.: PT-symmetry and antisymmetry by anti-Hermitian wave coupling and nonlinear optical interactions. Opt. Lett. 43, 4025-4028(2018)

[17] H, Chen., S, Hu.: The asymmetric solitons in two-dimensional parity-time-symmetric potentials. Phys. Lett. A 380, 162-165 (2016)

[18] Hxla, C., Yhd, A., Sjs, B., et al.: Two dimension PT-symmetry spacial soliton in atomic gases with linear and nonlinear potentials - ScienceDirect. Optik. 213, 164705 (2020)

[19] Zhang, Z., Wang, Y. P. Wang, X.: PT-symmetry-breaking-enhanced cavity optomechanical magnetometry. Phys. Rev. A 102, 023512 (2020)

[20] Xu, Z., Chen, S.: Dynamical evolution in a one-dimensional incommensurate lattice with PT symmetry. Phys. Rev. A 103, 043325 (2021)

[21] Longhi, S.: Quantum statistical signature of PT symmetry breaking. Opt. Lett. 45, 1591-1594 (2019)

[22] Nixon, S., Yang, J.: Nonlinear light behaviors near phase transition in non-parity-timesymmetric complex waveguides. Opt. Lett. 41, 2747-2750(2016)

[23] Fang, Y., Wu, G. Z., Wang, Y. Y., Dai, C.Q.: Data-driven femtosecond optical soliton excitations and parameters discovery of the high-order NLSE using the PINN. Nonlinear Dynamics. https://doi.org/10.1007/s11071-021-06550-9

[24] Yang, J.: Nonlinear waves in integrable and nonintegrable systems. Society for Industrial and Applied Mathematics(2010)

[25] Yang, J.: Can Parity-Time-Symmetric Potentials Support Families of Non-Parity-TimeSymmetric Solitons? Studies in Applied Mathematics.132, 332-353 (2014)

[26] Li, P., Dai, C., Li, R., Y, Gao.: Symmetric and asymmetric solitons supported by a PT-symmetric potential with saturable nonlinearity: bifurcation, stability and dynamics. Opt. Express. 26, 6949 (2018)

[27] Wu, Z., Cao, S., Che, W., F, Yang., X, Zhu., Y, He.: Solitons supported by parity-timesymmetric optical lattices with saturable nonlinearity in fractional Schrödinger equation. Results in Physics. 19, 103381 (2020) 
[28] Li, P., Dai, C.: Double Loops and Pitchfork Symmetry Breaking Bifurcations of Optical Solitons in Nonlinear Fractional Schrödinger Equation with Competing Cubic-Quintic Nonlinearities. Annalen der Physik. 532,2000048 (2020)

[29] Dong, L., Huang, C., Qi, W.: Symmetry breaking and restoration of symmetric solitons in partially parity-time-symmetric potentials. Nonlinear Dynamics. 98, 1701-1708 (2019)

[30] Chen, Yi. Xiang.: One-dimensional optical solitons in cubic-quintic-septimal media with PT symmetric potentials. Nonlinear Dynamics. 87, 1629-1635 (2017)

[31] Hanif, Y., Saleem, U.: Broken and unbroken PT -symmetric solutions of semi-discrete nonlocal nonlinear Schrödinger equation. Nonlinear Dynamics. 98, 233-244 (2019)

[32] Achilleos, V., Kevrekidis, P. G., Frantzeskakis, D. J., Carretero-Gonzalez, R.: Dark solitons and vortices in PT-symmetric nonlinear media: From spontaneous symmetry breaking to nonlinear PT phase transitions. Phys. Rev. A 86, 1-7 (2012)

[33] Yang, J., Stability analysis for pitchfork bifurcations of solitary waves in generalized NLSEs. Phys. D 244, 50-67 (2013) 